Revue

Revue de l'histoire des religions

de l'histoire des religions

$2 \mid 2006$

Varia

\title{
Le judaïsme caché du carré SATOR de Pompéi
}

The hidden judaism of the Pompeiian SATOR square

\section{Nicolas Vinel}

\section{OpenEdition}

\section{Journals}

Édition électronique

URL : http://journals.openedition.org/rhr/5136

DOI : 10.4000/rhr.5136

ISSN : 2105-2573

Éditeur

Armand Colin

Édition imprimée

Date de publication : 1 juin 2006

Pagination : 173-194

ISBN : 2200-92104-7

ISSN : 0035-1423

\section{Référence électronique}

Nicolas Vinel, «Le judaïsme caché du carré sator de Pompéi », Revue de l'histoire des religions [En ligne], 2 | 2006, mis en ligne le 19 janvier 2010, consulté le 10 décembre 2020. URL : http://

journals.openedition.org/rhr/5136 ; DOI : https://doi.org/10.4000/rhr.5136 


\section{NICOLAS VINEL \\ Centre Philosophies et Rationalités \\ Université de Clermont-Ferrand \\ Le judaïsme caché du carré SATOR de Pompéi}

Une découverte récente dans le domaine des mathématiques pythagoriciennes conduit au déchiffrement du carré SATOR, dont le plus ancien exemple est à Pompéi. Il cache une autre inscription en latin, et en toutes lettres, qui prouve que c'est un cryptogramme juif, fondé sur l'autel de bronze d'Ex 27 et le serpent de bronze de $\mathrm{Nb} 21$, comme signe de reconnaissance et symbole sotériologique pour la Diaspora. En outre, le mot «SAUTRAN » inscrit sous le SATOR à Pompéi n'est pas le salut d'un certain Sautran(us) : devant la lettre $N$, qui symbolise le serpent dans l'épigraphie judéo-chrétienne, SAUTRA transcrit un impératif de la racine hébraïque str «cacher», dont l'infinitif absolu est SATOR

\section{The hidden judaism of the Pompeiian SATOR square}

A recent discovery in the Pythagorean mathematical field leads to the deciphering of the SATOR square, the earliest examples of which are in Pompeii. It hides another Latin inscription, in full, which shows that it's a Jewish cryptogram, based on the bronze altar in Ex 27 and the bronze serpent in $\mathrm{Nb} 21$, as an identification mark and a symbol of salvation for the Diaspora. Furthermore, the word "SAUTRAN" below the SATOR in Pompeii doesn't mean the greeting from someone called Sautran(us) : before the letter $N$, symbol of the serpent according to the Judeo-Christian epigraphy, SAUTRA transcribes an imperative of the hebrew root str "to hide", the infinitive absolute of which is SATOR. 


\section{$\mathrm{R} O \mathrm{O}$ A S \\ O P E R A \\ $T$ E N E T \\ A R E P O \\ S A T O R}

Depuis la fin du XIX ${ }^{\mathrm{e}}$ siècle, le carré SATOR a suscité des hypothèses nombreuses et, disons-le, parfois peu raisonnables, mais dont le nombre et les excès mêmes mesurent la fortune épigraphique de ce palindrome à l'aube de l'ère chrétienne : au $\mathrm{III}^{\mathrm{e}}$ siècle en Angleterre et en Syrie, à Doura-Europos ; au début du II $^{\mathrm{e}}$ en Hongrie ; enfin, et surtout, au Ier à Pompéi. Rappelons quelques faits. Dans l'histoire de l'exégèse du SATOR, deux « découvertes » ont fait date, à dix ans d'intervalle. En 1926, quand le plus ancien SATOR connu datait du III ${ }^{\mathrm{e}}$ siècle, F. Grossser observa que le palindrome était composé des mêmes lettres que les deux mots Pater noster et montra qu'il était possible, avec ses vingt-cinq lettres, de former la croix suivante ${ }^{1}$ :

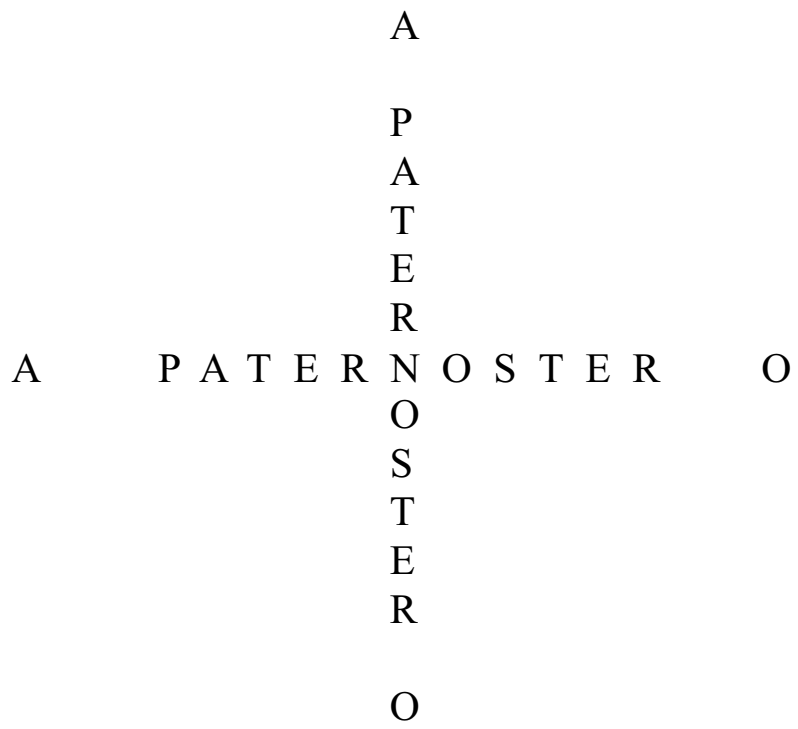

1. Felix Grosser, «Ein neuer Vorschlag zur Deutung der Satorformel », Archiv für Religionswissenschaft, 29, 1926, p. 165-169. 
Nombre d'exégètes prirent sur cette anagramme une entière assurance : elle ne pouvait être fortuite et constituait la preuve de l'origine chrétienne du carré. Or, l'année 1936 vit la découverte d'un SATOR à Pompéi, sur une colonne de la palestre, où il est complété par d'autres inscriptions : la même main a écrit au-dessous SAUTRAN puis, en caractères plus petits, VALE ; une seconde main a ajouté $\triangle$ au-dessus et $A N O$ au-dessous ; ce SATOR bien conservé permit à M. Della Corte de reconnaître le fragment d'un autre dans une inscription mutilée découverte en 1925, à l'intérieur d'une maison ${ }^{2}$. Antérieurs à l'éruption du Vésuve, le 24 août 79, ce sont à ce jour les plus anciens exemples connus du palindrome. On soupçonna dès lors l'anagramme de Grosser d'être une pure coïncidence, et l'hypothèse chrétienne fut contestée par ceux qui jugeaient improbable, à cette date, la création d'un tel cryptogramme par des chrétiens, voire l'existence même d'une communauté chrétienne à Pompéi. L'hypothèse juive fut alors envisagée, car on possédait déjà plusieurs indices sérieux d'une présence juive ${ }^{3}$ (même si la recherche récente doute qu'elle ait été «nombreuse » ${ }^{4}$ ), mais aucun argument décisif ne fut apporté 5 . Beaucoup gardèrent donc leur foi en l'interprétation chrétienne de Grosser, dont l'anagramme reste communément répandue $^{6}$, bien qu'un article spirituel et magistral de Paul Veyne ait dissipé une erreur si charmante ${ }^{7}$.

2. Matteo Della Corte, «Il crittogramma del "Pater noster" rinvenuto a Pompei », Atti della Pontificia Academia Romana di Archeologia, 12, 1936, p. 397-400 (photographie p. 398).

3. Jean-Baptiste Frey, «Les Juifs à Pompéi », Revue Biblique, 42, 1933, p. 365-384.

4. Voir Giancarlo Lacerenza, «Per un riesame della presenza ebraica a Pompei », Materia giudaica, 6 (1), 2001, p. 99-103.

5. Status quaestionis par Duncan Fishwick, «On the origin of the rotas-sator square », Harvard Theological Review, 57, 1964, p. 39-53.

6. Jean Carmignac, Recherches sur le Notre Père, Paris, Letouzey, 1969, p. 448-468 ; Rino Cammilleri, Il quadrato magico. Un mistero che dura da duemila anni, BUR, Milan, 2004².

7. Paul Veyne, «Le carré Sator ou beaucoup de bruit pour rien », Bulletin de l'Association Guillaume Budé. Lettres d'Humanité, t. 27, 4e série, nº 4, décembre 1968, p. 427-456. 
Dans ces circonstances, les pages qui suivent n'auraient jamais vu le jour sans une découverte récente dans le domaine des mathématiques anciennes, au sujet des carrés que nous appelons aujourd'hui « magiques »; ceux-ci sont connus avant l'ère chrétienne en Chine et quelques siècles plus tard en Inde, mais on les croyait absents de la tradition gréco-romaine jusqu'au XIV $\mathrm{V}^{\mathrm{e}}$ siècle, où un opuscule byzantin leur est explicitement consacré ${ }^{8}$ (les interprètes du SATOR lui ont logiquement accordé peu d'intérêt, du fait de son caractère $\operatorname{tardif}^{9}$ ) ; or, comme on peut le lire dans la revue Archive for History of Exact Sciences, un passage du traité consacré par Jamblique (III ${ }^{\mathrm{e}}-\mathrm{IV}^{\mathrm{e}} \mathrm{s}$.) à l'arithmétique pythagoricienne, à propos des carrés naturels et non naturels de trois, implique non seulement une connaissance minimale de ceux que nous appelons «magiques», mais permet aussi d'expliquer leur découverte ${ }^{10}$. Sachant que le berceau du pythagorisme est le sud de l'Italie ${ }^{11}$, ce locus ouvre d'emblée une perspective nouvelle à l'étude du SATOR de Pompéi : aurait-il été chiffré grâce à un carré pythagoricien de cinq ?

Cette perspective s'accorde avec l'hypothèse juive, car, au I ${ }^{\mathrm{er}}$ siècle, l'assimilation de l'arithmétique grecque et du pythagorisme par le judaïsme est attestée non seulement dans la Diaspora alexandrine, où Philon va jusqu'à associer le Tétragramme à la Tétraktys ${ }^{12}$, mais aussi dans le judaïsme palestinien, car « des lettres grecques furent utilisées pour désigner des nombres y compris dans le Temple même $(\text { Shek. } 3,2)^{13} »$ et Flavius Josèphe affirme sans ambages que les

8. Paul Tannery, «Le traité de Manuel Moschopoulos sur les carrés magiques. Texte grec et traduction », Mémoires scientifiques, t. IV, Paris, Gabay, 19962, p. 26-60.

9. Voir notamment Guillaume de Jerphanion, « La formule magique SATOR AREPO ou ROTAS OPERA. Vieilles théories et faits nouveaux », Recherches de Science Religieuse, 25, 1935, p. 214.

10. Nicolas Vinel, «Un carré magique pythagoricien ? », Archive for History of Exact Sciences, 59 (6), 2005, p. 545-562.

11. Voir Porphyre, Vita Pythagorae, éd. Nauck, Leipzig, Teubner, $1886^{2}$, p. $18-20$.

12. Philon, De vita Mosis, II, 115, éd. Arnaldez, Cerf, Paris, 1967, p. 242.

13. Gershom Scholem, La Kabbale, Gallimard, Paris, $2003^{3}$, p. 511. 
Esséniens ont adopté le mode de vie enseigné par Pythagore ${ }^{14}$ (selon les mots de Martin Hengel, « the essential thing is not the supposed "Pythagorean" influences, but the fact that Hellenistic observers like Josephus [...] could present them as Jewish "Pythagoreans" 15 »); à cela s'ajoute le thème inverse du « larcin des Grecs » et de l'origine hébraïque du pythagorisme, topos littéraire révélateur, lui aussi, de son assimilation par le judaïsme hellénisé ${ }^{16}$.

C'est pourquoi l'hypothèse d'un cryptogramme juif utilisant l'arithmétique pythagoricienne est légitime. Sa validité sera établie par le déchiffrement du SATOR, qui s'avère à la fois un signe de reconnaissance juif, aux dimensions de l'autel de bronze d'Ex 27, et un symbole sotériologique, image du serpent de bronze de $\mathrm{Nb} 21$; ce symbole est souligné par SAUTRAN, transcription d'un impératif hébraïque précédant la lettre $N$, symbole du serpent dans l'épigraphie judéo-chrétienne.

\section{ARA AEREA : UN TÉMOIN POUR LA DIASPORA}

La tradition biblique relative à l'autel des holocaustes atteste qu'il possède, outre sa fonction liturgique, une importante valeur symbolique. Dans le livre de Josué, notamment, la Diaspora transjordanienne est confrontée à la problématique de l'exil : peut-on servir YHWH en terre étrangère ? Ayant bâti une reproduction de l'autel de YHWH, les tribus transjordaniennes répondent en ces termes à l'accusation d'infidélité à son culte (Jos 22,28-29) :

« Regardez la forme de l'autel de YHWH que nos pères ont fait, non en vue d'holocaustes ou d'autres sacrifices, mais comme un témoin entre nous et vous. Loin de nous de nous révolter contre YHWH et de nous détourner aujourd'hui de YHWH en bâtissant, pour y offrir

14. Flavius Josèphe, Antiquitates Judaicae, XV, 371. Dernier état de la recherche dans Justin Taylor, Pythagoreans and Essenes. Structural parallels (« Collection de la Revue des Études Juives», 32), Peeters, Paris-Louvain, 2004.

15. Martin Hengel, Judaism and Hellenism: Studies in their Encounter in Palestine during Early Hellenistic Period, Fortress, Philadelphia, 1974, t. I, p. 247.

16. Voir Peter Gorman, «Pythagoras Palaestinus », Philologus, 127, 1983, p. $30-42$. 
holocaustes, oblations ou sacrifices, un autel rival de l'autel de YHWH notre Dieu, érigé devant sa demeure. ${ }^{17}$ »

C'est donc dans sa «forme » que réside la fonction symbolique de l'autel. Or, dans le livre de l'Exode, l'autel de bronze construit par Beçaléel a une « forme » très particulière, conforme aux instructions divines reçues par Moïse en Ex 27,1-2 :

«Tu feras l'autel en bois d'acacia; de cinq coudées de long et de cinq coudées de large, l'autel sera carré ; il aura trois coudées de haut. Tu feras à ses quatre angles des cornes faisant corps avec lui, et tu le plaqueras de bronze. »

La valeur symbolique de ses dimensions est attestée dans le livre des Chroniques (IV ${ }^{\mathrm{e}}-\mathrm{III}^{\mathrm{e}} \mathrm{s}$.), dont les chapitres 2 Ch 1-9 racontent la construction du temple de Jérusalem par Salomon. Celui-ci se rend tout d'abord au lieu de culte de Gabaon, où se trouve la Tente de la Rencontre : «L'autel de bronze qu'avait fait Beçaléel, fils de Uri, fils de Hur, était là devant la Demeure de YHWH » (2 Ch 1,5). De retour à Jérusalem, Salomon ordonne de bâtir un temple, mais choisit pour l'autel des holocaustes des dimensions plus imposantes : « Il fit un autel de bronze, long de vingt coudées, large de vingt et haut de $\operatorname{dix}^{18} \gg(2 \mathrm{Ch} 4,1)$; quant à celui d'Ex 27 , il est pris comme modèle d'un socle placé sur le parvis du temple (2 Ch 6,13) :

«Salomon avait fait un socle de bronze qu'il avait mis au milieu de la cour ; il avait cinq coudées de long, cinq de large et trois de haut. Salomon y monta, s'y tint et s'y agenouilla en présence de toute l'assemblée d'Israël. »

$\mathrm{Vu}$ la correspondance de ces dimensions avec celles du SATOR, on peut se demander si elles avaient encore au $\mathrm{I}^{\mathrm{er}}$ siècle la valeur symbolique qu'elles ont pour le Chroniste, trois siècles plus tôt. En d'autres termes, la «forme » de l'autel d'Ex 27 était-elle pour la Diaspora méditerranéenne ce qu'est la « forme » de l'autel de Jos 22 pour la Diaspora transjordanienne : un signe de reconnaissance entre fidèles de YHWH ?

17. Les traductions sont celles de la Bible de Jérusalem, excepté pour le Tétragramme transcrit YHWH et nehochet toujours traduit par «bronze ». petit. »

18. $C f .1 \mathrm{R} 8,64$ : "L'autel de bronze qui était devant YHWH était trop 
En effet, le Sator de Pompéi s'inscrit dans une période où les juifs ont dû quitter Rome à deux reprises : sous Tibère en l'an 19 et sous Claude en l'an 49, comme en témoignent les Actes des Apôtres ${ }^{19}$. Il est donc vraisemblable que certains aient utilisé un tel signe de reconnaissance à l'insu des païens : les dimensions de l'autel de bronze constituaient une base particulièrement pertinente pour un cryptogramme, visible même une fois le carré chiffré. Grâce à quel chiffre? Le locus pythagoricus de Jamblique permet de supposer que c'est un carré non naturel de 5 respectant deux propriétés : ses lignes et colonnes ont une somme égale à $65 ; 13$, au centre du carré, est la moyenne arithmétique des couples de nombres pris symétriquement autour de lui, donc égaux à $26(13+26+26=65)$. C'est le cas du carré transmis par l'opuscule byzantin ${ }^{20}$ :

65 \begin{tabular}{c|c|c|c|c|c|}
65 & 65 & 65 & 65 & 65 & 65 \\
\hline 11 & 24 & 7 & 20 & 3 & 65 \\
\cline { 1 - 5 } & 12 & 25 & 8 & 16 & 65 \\
\hline 17 & 5 & 13 & 21 & 9 & 65 \\
\cline { 1 - 5 } 10 & 18 & 1 & 14 & 22 & 65 \\
\cline { 1 - 4 } 23 & 6 & 19 & 2 & 15 & 65
\end{tabular}

Dans l'hypothèse où le SATOR a une origine juive, un tel carré ne manque pas d'intérêt, puisque les nombres 13, 26 et 65 sont connus pour signifier le nom divin dans la gématrie hébraïque : 26 est la valeur numérique du Tétragramme, 65 celle de sa forme lue «Adonaï », 13 celle de ehad «Un» (Dt 6,4). Or la pratique de la gématrie, ou isopséphie, est attestée à Pompéi par plusieurs inscriptions grecques ${ }^{21}$ :

$-\mathrm{n}^{\circ} 12^{*}:$ «J'aime celle dont le chiffre (arithmos) est (...) 1.»

19. Ac 18,2 : «Un édit de Claude ordonnait à tous les juifs de s'éloigner de Rome. » Voir Leonard Victor Rutgers, « Roman Policy towards the Jews: Expulsions from the City of Rome during the First Century C.E. », Classical Antiquity, 13, 1994, p. 56-74, repris dans The Hidden Heritage of Diaspora Judaism, Peeters, Louvain, 1998, p. 171-191.

20. Paul Tannery, « Le traité de Manuel Moschopoulos », art. cit., p. 54.

21. Corpus Inscriptionum Latinarum, t. IV, Suppl., Berlin, 1901, p. 460, 557 sq. 
$-\mathrm{n}^{\circ} 4839$ : « celle dont 1035 est le chiffre du beau nom (arithmos tou kalou onomatos) »

$-\mathrm{n}^{\circ} 4861$ : «J'aime celle dont 545 est le chiffre (arithmos). »

Très tôt, on a souligné le parallèle entre ces inscriptions de Pompéi et le texte contemporain de l'Apocalypse sur le chiffre de la Bête ${ }^{22}$ :

- Ap 13,17 : « le nom de la Bête ou le chiffre de son nom (ton arithmon tou onomatos) »

- Ap 13,18 : « Son chiffre (arithmos), c'est 666. »

$\mathrm{Au}$ vu de ces parallèles contemporains du SATOR et de nombres tels que 1035 ou 666, une isopséphie 65 pour « Adonaï » dans le carré pompéien n'a rien d'invraisemblable. Certes, les spéculations de ce genre nous rebutent aujourd'hui, et souvent à bon droit, mais il faut savoir que même dans la tradition chinoise, où la connaissance du carré magique de trois est certaine avant l'ère chrétienne, le carré lui-même n'est attesté explicitement qu'au $\mathrm{X}^{\mathrm{e}}$ siècle, « after some thirteen hundred years of hidden, private or cultic use ${ }^{23} »$. Il est donc possible que des raisons religieuses ou ésotériques expliquent un aussi long silence entre le SATOR et le traité byzantin.

Gershom Scholem souligne une telle tradition ésotérique liée au pythagorisme dans le Sefer Yezira, le plus ancien texte connu de la mystique juive $\left(\mathrm{III}^{\mathrm{e}}-\mathrm{VI}^{\mathrm{e}} \mathrm{s}\right.$.), pour sa conception de la Création à partir de lettres et de nombres, qui « tire probablement son origine de sources néo-pythagoriciennes ${ }^{24} »$. Or la correspondance entre lettres et nombres est justement au cœur du cryptogramme de Pompéi, notamment parce que son centre $N, 13^{\mathrm{e}}$ lettre des alphabets latin et grec (et qui vaut 13 par exemple sur des pièces grecques du $\mathrm{II}^{\mathrm{e}}$ siècle av. J.-C. ${ }^{25}$ ), coïncide avec le centre du carré naturel de cinq, mais

22. Antonio Sogliano, «Isopsepha Pompeiana », Rendiconti della Reale Academia dei Lincei, 10, 1901, p. 256-259.

23. Steve Cammann, « The magic square of Three in old chinese philosophy and religion », History of Religions, 1, 1960, p. 37-80 (p. 45).

24. Gershom Scholem, Les origines de la Kabbale, Aubier, Paris, 1966, p. 37.

25. Otto Neugebauer, The exact sciences in Antiquity, New York, 19572, p. 9 : « The months of issue are denoted by the letters A to M representing the numbers 1 to 12 for an ordinary year, adding $\mathrm{N}=13$ for a leap year. » 
surtout parce qu'il suffit de transformer ce carré naturel en carré «Adonaï » pour déchiffrer le carré SATOR.

1) La croix centrale subit une rotation de $45^{\circ}$ à droite :

\begin{tabular}{|c|c|c|c|c|}
\hline 1 & 2 & 3 & 4 & 5 \\
\hline 6 & 7 & 8 & 9 & 10 \\
\hline 11 & 12 & 13 & 14 & 15 \\
\hline 16 & 17 & 18 & 19 & 20 \\
\hline 21 & 22 & 23 & 24 & 25 \\
\hline
\end{tabular}

\begin{tabular}{|c|c|c|c|c|}
\hline 11 & 24 & 7 & 20 & 3 \\
\hline 4 & 12 & 25 & 8 & 16 \\
\hline 17 & 5 & 13 & 21 & 9 \\
\hline 10 & 18 & 1 & 14 & 22 \\
\hline 23 & 6 & 19 & 2 & 15 \\
\hline
\end{tabular}

\begin{tabular}{|c|c|c|c|c|}
\hline R & O & T & A & S \\
\hline O & P & E & R & A \\
\hline T & E & N & E & T \\
\hline A & R & E & P & O \\
\hline S & A & T & O & R \\
\hline
\end{tabular}

\begin{tabular}{|c|c|c|c|c|}
\hline $\mathrm{T}$ & & & & $\mathrm{T}$ \\
\hline & $\mathrm{E}$ & & $\mathrm{E}$ & \\
\hline & & $\mathrm{N}$ & & \\
\hline & $\mathrm{E}$ & & $\mathrm{E}$ & \\
\hline $\mathrm{T}$ & & & & $\mathrm{T}$ \\
\hline
\end{tabular}

2) La croix qui relie les angles opère la même rotation, mais les paires de nombres placées symétriquement autour de 13, leur moyenne arithmétique (1-25, 5-21, 7-19, 9-17), passent de l'extérieur à l'intérieur, et vice-versa :

\begin{tabular}{|c|c|c|c|c|}
\hline 1 & 2 & 3 & 4 & 5 \\
\hline 6 & 7 & 8 & 9 & 10 \\
\hline 11 & 12 & 13 & 14 & 15 \\
\hline 16 & 17 & 18 & 19 & 20 \\
\hline 21 & 22 & 23 & 24 & 25 \\
\hline
\end{tabular}

\begin{tabular}{|c|c|c|c|c|}
\hline 11 & 24 & 7 & 20 & 3 \\
\hline 4 & 12 & 25 & 8 & 16 \\
\hline 17 & 5 & 13 & 21 & 9 \\
\hline 10 & 18 & 1 & 14 & 22 \\
\hline 23 & 6 & 19 & 2 & 15 \\
\hline
\end{tabular}

\begin{tabular}{|c|c|c|c|c|}
\hline $\mathrm{R}$ & $\mathrm{O}$ & $\mathrm{T}$ & $\mathrm{A}$ & $\mathrm{S}$ \\
\hline $\mathrm{O}$ & $\mathrm{P}$ & $\mathrm{E}$ & $\mathrm{R}$ & $\mathrm{A}$ \\
\hline $\mathrm{T}$ & $\mathrm{E}$ & $\mathrm{N}$ & $\mathrm{E}$ & $\mathrm{T}$ \\
\hline $\mathrm{A}$ & $\mathrm{R}$ & $\mathrm{E}$ & $\mathrm{P}$ & $\mathrm{O}$ \\
\hline $\mathrm{S}$ & $\mathrm{A}$ & $\mathrm{T}$ & $\mathrm{O}$ & $\mathrm{R}$ \\
\hline
\end{tabular}

\begin{tabular}{|l|l|l|l|l|}
\hline & & P & & \\
\hline & & R & & \\
\hline R & S & N & S & R \\
\hline & & R & & \\
\hline & & P & & \\
\hline
\end{tabular}


3) Restent huit nombres pairs qui correspondent aux A et $\mathrm{O}$; ils permutent simplement entre eux :

\begin{tabular}{|c|c|c|c|c|}
\hline R & O & T & A & S \\
\hline O & P & E & R & A \\
\hline T & E & N & E & T \\
\hline A & R & E & P & O \\
\hline S & A & T & O & R \\
\hline
\end{tabular}

\begin{tabular}{|l|l|l|l|l|}
\hline & $\mathrm{O}$ & & $\mathrm{O}$ & \\
\hline $\mathrm{A}$ & & & & $\mathrm{A}$ \\
\hline & & & & \\
\hline $\mathrm{A}$ & & & & $\mathrm{A}$ \\
\hline & $\mathrm{O}$ & & $\mathrm{O}$ & \\
\hline
\end{tabular}

Ainsi déchiffré par le carré « Adonaï », le cryptogramme contient, outre les deux TENET, les mots latins ARA AEREA « autel de bronze $^{26} \gg$ :

\begin{tabular}{|c|c|c|c|c|}
\hline $\mathrm{T}$ & & & & $\mathrm{T}$ \\
\hline & $\mathrm{E}$ & & $\mathrm{E}$ & \\
\hline & & $\mathrm{N}$ & & \\
\hline & $\mathrm{E}$ & & $\mathrm{E}$ & \\
\hline $\mathrm{T}$ & & & & $\mathrm{T}$ \\
\hline
\end{tabular}

\begin{tabular}{|c|c|c|c|c|}
\hline $\mathrm{T}$ & $\mathrm{O}$ & $\mathrm{P}$ & $\mathrm{O}$ & $\mathrm{T}$ \\
\hline $\mathrm{A}$ & $\mathrm{E}$ & $\mathrm{R}$ & $\mathrm{E}$ & $\mathrm{A}$ \\
\hline $\mathrm{R}$ & $\mathrm{S}$ & $\mathrm{N}$ & $\mathrm{S}$ & $\mathrm{R}$ \\
\hline $\mathrm{A}$ & $\mathrm{E}$ & $\mathrm{R}$ & $\mathrm{E}$ & $\mathrm{A}$ \\
\hline $\mathrm{T}$ & $\mathrm{O}$ & $\mathrm{P}$ & $\mathrm{O}$ & $\mathrm{T}$ \\
\hline
\end{tabular}

\begin{tabular}{|c|c|c|c|c|}
\hline & & & & \\
\hline $\mathrm{A}$ & $\mathrm{E}$ & $\mathrm{R}$ & $\mathrm{E}$ & $\mathrm{A}$ \\
\hline $\mathrm{R}$ & & & & $\mathrm{R}$ \\
\hline $\mathrm{A}$ & $\mathrm{E}$ & $\mathrm{R}$ & $\mathrm{E}$ & $\mathrm{A}$ \\
\hline & & & & \\
\hline
\end{tabular}

Il est crucial de noter la correspondance entre ce carré $A R A$ AEREA et les instructions divines d'Ex 27,1-2 pour l'autel de bronze : «De cinq coudées de long et de cinq coudées de large, l'autel sera carré $[. .$.$] . Tu feras à ses quatre angles des cornes faisant corps avec$ lui, et tu le plaqueras de bronze. » À chaque «corne » correspond un $T$, dont Tertullien ( $\mathrm{II}^{\mathrm{e}} \mathrm{s}$.) atteste qu'il est la transcription latine du $\operatorname{taw}^{27}$, ce « signe » que Dieu ordonne d'inscrire sur le front des justes en $\mathrm{Ez} 9,4$; or cette scène se déroule «devant l'autel de bronze » (Ez 9,2) ; en outre, les cornes sont la partie la plus sainte de l'autel, si bien que l'on ne pouvait porter la main sur celui qui les saisissait : TENET exprime probablement cette idée dans le carré ARA AEREA, comme plus tard la Vulgate de $1 \mathrm{R}$ 1,50, tenuit cornu altaris « il saisit une corne de l'autel ».

26. Le nom ara et l'adjectif aereus sont courants dans le latin du $\mathrm{I}^{\mathrm{er}}$ siècle ; la Vulgate choisira la traduction plus rare altare aeneum (Ex 38,30 ; 39,39; 2 R 16,14 , etc.).

27. Tertullien, Adversus Marcionem, III, 22 (PL, éd. Migne, t. II, col. 353) : "Da signum TAU in frontibus virorum". Ipsa est enim littera Gracorum Tau, nostra autem T. " "Mets le signe TAU sur le front des hommes"; c'est le Tau des Grecs, et notre T. » 
Le SATOR montre donc que la « forme » de l'autel des holocaustes avait toujours, à l'aube de l'ère chrétienne, la valeur symbolique que lui attribue le Chroniste au Ive siècle, et qu'elle restait pour la Diaspora méditerranéenne ce qu'elle est pour la Diaspora transjordanienne de Jos 22 : « un témoin ('ed) entre nous que c'est YHWH qui est Dieu » (v. 34). Toutefois, ce n'est pas sa seule valeur.

\section{SERPENS AEREA : UN SYMBOLE SOTÉRIOLOGIQUE}

Outre cette fonction de témoin, le SATOR n'avait-il pas une valeur sotériologique, au sens où le salut, «c'est échapper à quelque mal physique, au danger, aux mains d'un ennemi ${ }^{28}$ » ? En effet, selon Isaïe, l'autel témoin annonce la délivrance future de la Diaspora (Is 19,19-20) :

«Ce jour-là, il y aura un autel dédié à YHWH au milieu du pays d'Égypte [...]. Ce sera un signe et un témoin ('ed) de YHWH Sabaot au pays d'Égypte. Quand ils crieront vers YHWH par crainte des oppresseurs, il leur enverra un sauveur et un défenseur qui les délivrera. »

Or le bronze est la matière d'un autre symbole de salut, plus célèbre encore, à savoir le serpent fabriqué par Moïse en Nb 21,6-9 :

«Dieu envoya contre le peuple les serpents brûlants, dont la morsure fit périr beaucoup de monde en Israël. Le peuple vint dire à Moïse : "Nous avons péché en parlant contre YHWH et contre toi. Intercède auprès de YHWH pour qu'il éloigne de nous ces serpents." Moïse intercéda pour le peuple et YHWH lui répondit : "Façonne-toi un Brûlant que tu placeras sur un étendard (nes, sèmeion LXX). Quiconque aura été mordu et le regardera restera en vie." Moïse façonna donc un serpent de bronze qu'il plaça sur l'étendard (nes), et si un homme était mordu par quelque serpent, il regardait le serpent de bronze et restait en vie. »

L'importance extrême de cet épisode pour la Diaspora alexandrine contemporaine du SATOR est attestée par le livre de la Sagesse ( ${ }^{\mathrm{er}} \mathrm{s}$. av.), qui fait du serpent de $\mathrm{Nb} 21$ le symbolon de Dieu lui-même (Sg 16,5-7) :

«Même lorsque s'abattit sur eux la fureur terrible de bêtes féroces, et qu'ils périssaient sous les morsures de serpents tortueux, ta colère ne

28. Marcel Simon, «Conceptions et symboles sotériologiques chez les juifs de la Diaspora », in U. Bianchi et M.J. Vermaseren, La soteriologia dei culti orientali nell'impero romano, Brill, Leiden, 1982, p. 781-802 (p. 781). 
dura pas jusqu'au bout; mais c'est par manière d'avertissement et pour peu de temps qu'ils furent inquiétés, et ils avaient un signe (sumbolon) de salut pour leur rappeler le commandement de ta Loi, car celui qui se tournait vers lui était sauvé, non par ce qu'il avait sous les yeux, mais par toi, le Sauveur de tous. »

De même, dans son ouvrage sur les symboles juifs de la période gréco-romaine, Goodenough ${ }^{29}$ répertorie une amulette grecque qui porte d'un côté l'inscription MOYCH « Moïse » et, de l'autre, le dessin d'un serpent entre les mots IA $\Omega^{30}$ «YHWH » et CABA $\Omega$ «Sabaot» (cf. Is 19,20).

Une fois de plus, déchiffré par le carré «Adonaï», le SATOR s'inscrit dans cette tradition, car ARA et AEREA sont accompagnés par le nom SERPENS $S^{31}$ - l'ensemble devant être scellé par la dernière pièce manquante du carré :

\begin{tabular}{|c|c|c|c|c|}
\hline & & P & & \\
\hline A & E & R & E & A \\
\hline R & S & N & S & R \\
\hline A & E & R & E & A \\
\hline & & P & & \\
\hline
\end{tabular}
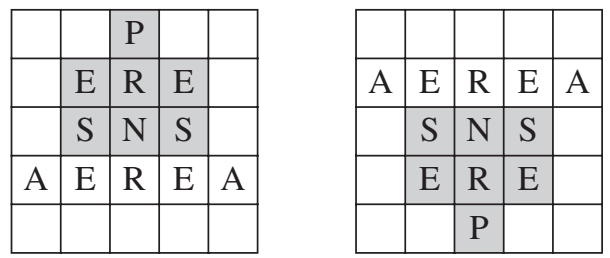

La synthèse de l'autel de bronze et du serpent de $\mathrm{Nb} 21$ est corroborée par Genèse Rabba, qui souligne la parenté entre les noms hébraïques du bronze - ou airain - et du serpent :

«Le Saint, béni soit-Il, ordonna à Moïse : "Fais-toi un serpent (saraf)" sans préciser davantage. Et Moïse de dire : "Si je le fais en or (zahav), les deux mots ne correspondront pas, en argent (keseph), les deux mots ne correspondront pas non plus. Je vais donc le faire en airain, et là les deux mots correspondront." Moïse fit le serpent (nahach) avec de l'airain (nehochet)..$^{32} »$

29. Erwin R. Goodenough, Jewish Symbols in the Greco-Roman Period, t. III, New York, 1953, ill. ${ }^{\circ} 1135$.

30. Diodore de Sicile ( ${ }^{\mathrm{er}}$ s. av. J.-C.) associe à Moïse le «dieu appelé Iao » (Bibliotheca historica, I, 94, 2, éd. Vogel, Teubner, Leipzig, 1888).

31. Sur le féminin SERPENS AEREA, voir Ernout-Meillet, Dictionnaire étymologique de la langue latine, Klincksieck, Paris, 1994 , p. 619 : «serpens f. (scil. bestia)».

32. Midrach Rabba, t. I, 31, 8, trad. Maruani et Cohen-Arazi, Verdier, 1987, p. 322. 
Ce midrash est vraisemblablement aussi ancien que le nom Nehouchtân, donné au serpent de bronze de Moïse quand, selon le livre des Rois, il était honoré dans le Temple ${ }^{33}$. En Nb 21 même, selon M. Morgen, si le rédacteur présente l'épisode comme un événement voulu par Dieu, c'est précisément «pour expliquer la présence de cette croyance aux pouvoirs prophylactiques du serpent d'airain qui a eu une certaine importance en Israël (II R 18,4) et ne pas la confondre avec un culte idolâtrique ${ }^{34} \gg$. Le SATOR déchiffré s'inscrit dans cette tradition, puisqu'il s'avère à la fois $A R A A E R E A$ (nehochet) par ses dimensions et ARA SERPENS (nahach) par sa structure interne.

En effet, une fois de plus, la correspondance entre le fond et la forme du cryptogramme est cruciale : de même que la mention $A R A$ AEREA correspond aux dimensions du carré, SERPENS montre que le palindrome imite la forme du serpent (tel l' " amphisbène ", ce serpent auquel les Grecs attribuaient deux têtes et la possibilité de se mouvoir en avant comme en arrière); SERPENS correspond aussi à la propriété d'être lu boustrophédon (alternativement de gauche à droite et de droite à gauche), qui trouve un parallèle remarquable à Pompéi dans le serpentis lusus : ces deux mots latins, qui signifient le «jeu du serpent», sont l'incipit d'une longue inscription qui offre un bel exemple de calligramme ; en effet, son texte écrit verticalement dessine un serpent à quatre boucles, analogue au SATOR ci-dessous $^{35}$ :

$$
\begin{array}{|l|ll|ll|}
\cline { 3 - 5 } & \mathrm{O} & \mathrm{T} & \mathrm{A} & \mathrm{S} \\
\mathrm{O} & \mathrm{P} & \mathrm{E} & \mathrm{R} & \mathrm{A} \\
\mathrm{T} & \mathrm{E} & \mathrm{N} & \mathrm{E} & \mathrm{T} \\
\mathrm{A} & \mathrm{R} & \mathrm{E} & \mathrm{P} & \mathrm{O} \\
\mathrm{S} & \mathrm{A} & \mathrm{T} & \mathrm{O} & \mathrm{R} \\
\hline
\end{array}
$$

33. 2 R 18,4 : «Il mit en pièces le serpent de bronze que Moïse avait fabriqué. Jusqu'à ce temps-là, en effet, les Israélites lui offraient des sacrifices ; on l'appelait Nehushtân. »

34. Michèle Morgen, «Le Fils de l'Homme élevé en vue de la vie éternelle », Revue des Sciences Religieuses, 68 (1), 1994, p. 9.

35. C.I.L., t. IV, Berlin, 1871, $\mathrm{n}^{\circ} 1595$. 
Néanmoins, contrairement au serpentis lusus, le SATOR n'a ni début ni fin et revient toujours au point d'où il est parti - l'exact contraire des impies dans le livre de la Sagesse : « Notre fin est sans retour, le sceau est apposé et nul ne revient » $(\mathrm{Sg} 2,5)$. C'est pourquoi il est SERPENS AEREA, car le serpent de $\mathrm{Nb} 21$ est au $\mathrm{I}^{\mathrm{er}}$ siècle un symbole d'éternité et de résurrection, attesté non seulement en $\mathrm{Sg} 16$ (supra), mais aussi dans l'Évangile de Jean : «Comme Moïse éleva le serpent dans le désert, ainsi faut-il que soit élevé le Fils de l'homme, afin que quiconque croit ait en lui la vie éternelle » (Jn 3,14-15).

C'est le moment de commenter les inscriptions de la seconde main au-dessus et au-dessous du SATOR de la palestre : $\Delta$ et $A N O$. Selon E. Testa, les ossuaires judéo-chrétiens associent la lettre $N$ à des dessins de serpents et le serpent lui-même apparaît dans les inscriptions du Dominus Flevit « sulla forma sinuosa di una $N »^{36}$; ce symbolisme remonte à l'origine même des alphabets grec et hébreu, car le signe pictographique dont proviennent les lettres $n u$ et nun était un serpent et, à ce titre, la lettre $N$ est aussi un symbole d'éternité (le C.I.L. relève près de Pompéi, à Naples, une inscription où un $N$ est isolé sous les mots AETERNO FLORE ${ }^{37}$ ). Or les ossuaires étudiés par E. Testa attestent aussi la lettre $\Delta$. Rien d'étonnant donc à ce qu'une seconde main ait inscrit à la fois $\Delta$ au-dessus du SATOR et $A N O$ au-dessous, le symbole du serpent étant complété par les deux lettres qui symbolisent l'éternité (le même type de symbolisme apparaît sur une amulette répertoriée par Goodenough, où un serpent imposant est entouré par l'A et l' $\Omega$ du nom divin IA $\Omega^{38}$ ). Précisons que le symbolisme latin $\mathrm{AO}$ est bien attesté dans l'épigraphie judéochrétienne, à côté de l'hébreu aleph-taw et du grec alpha-oméga ${ }^{39}$ - ce dernier étant déjà utilisé par les Septante, au III ${ }^{\mathrm{e}}$ siècle $^{40}$.

36. Emanuele Testa, Il simbolismo dei Giudeo-Cristiani, Jérusalem, 1962 , p. 142 (oss. 15, 46, 123) et 161.

37. C.I.L., t. X, ${ }^{\circ} 362$ (cité par Testa, loc. cit.).

38. Erwin R. Goodenough, Jewish Symbols, op. cit., ill. $\mathrm{n}^{\circ}$ 1167. Cf. $\mathrm{n}^{\circ} 1135$ (supra), où le serpent est entouré par IA $\Omega$ et CABA $\Omega$ (déformation manifestement volontaire de Sabaot).

39. Emanuele Testa, op. cit., p. 347 et 364.

40. Voir Nicolas Vinel, «L'Alpha-Omega des Septante », Revue Biblique, 2006, t. 113 (en cours de parution). 
En définitive, la synthèse de l'autel de bronze et du serpent de $\mathrm{Nb} 21$ s'avère particulièrement cohérente, notamment du point de vue des sources bibliques. En effet, l'épisode de $\mathrm{Nb} 21$ est précédé en $\mathrm{Nb} 17$ par une étiologie du bronze de l'autel des holocaustes, donné comme « signe » à Israël. C'est la pièce manquante du cryptogramme.

\section{ARA, AEREA, SERPENS : TroIS « SIGNES » POUR LA DiasPora}

Jos 22 montre que l'autel des holocaustes ne vaut pas seulement par sa fonction liturgique, mais aussi par sa valeur symbolique, comme témoin pour la Diaspora. De ce point de vue, en tant qu'image de l'autel de bronze, le SATOR s'accorde avec la conception de la Tente de la Rencontre comme «signe visible » de la présence de Dieu en dehors de la Terre Sainte ${ }^{41}$.

Or la notion de «signe » (hébreu 'ôt, grec sèmeion) définit les trois mots inscrits en latin dans le cryptogramme, à commencer par l'autel de YHWH, dans le passage d'Isaïe vu plus haut, à propos de la Diaspora opprimée : « Ce sera un signe ('ôt, sèmeion LXX) et un témoin de YHWH Sabaot au pays d'Égypte» (Is 19,20). En Nb 17,1-4, c'est au bronze même de l'autel que Dieu attribue cette fonction, après la querelle des encensoirs :

«YHWH parla à Moïse et dit: "[...] Ces encensoirs de péché sont sanctifiés, au prix de la vie de ces hommes. Puisqu'on les a apportés devant YHWH et qu'ils sont consacrés, qu'on en batte le métal en plaques pour recouvrir l'autel. Ils serviront de signe ('ôt, sèmeion LXX) aux Israélites." Éléazar, le prêtre, prit les encensoirs de bronze qu'avaient apportés les hommes que le feu avait détruits. On les battit en plaques pour recouvrir l'autel. »

Cette étiologie du bronze devait avoir beaucoup d'importance pour le judaïsme alexandrin, car les Septante l'insèrent en Ex 38,22, dans le récit de la construction de l'autel par Beçaléel : "Il fit l'autel de bronze avec les encensoirs de bronze appartenant aux hommes qui s'étaient révoltés avec la communauté de Koré. »

41. Voir Bernard Renaud, «La formation de Ex 19-40. Quelques points de repère », Le Pentateuque. Débats et recherches, Cerf, Paris, 1992 ( "Lectio Divina », 151), p. 106. 
En troisième lieu, 'ôt caractérise aussi le bâton de Moïse changé en serpent, "premier signe » pour qu'Israël ait confiance en son guide (Ex 4,2-8). S'agissant de $\mathrm{Nb} 21$, il y a le mot nes « enseigne », que la Septante traduit comme 'ôt, par sèmeion, et qui devient même symbolon en Sg 16 (supra) : selon la formule de M. Morgen, l'événement de $\mathrm{Nb} 21$ «prend valeur de signe ${ }^{42}$ »; du reste, cela n'est pas propre à la tradition alexandrine, car le Targum Onkelos de $\mathrm{Nb} 21$ traduit nes par ' $a t^{43}$, équivalent araméen de l'hébreu 'o $t^{44}$; il s'avère donc que, dans la réception juive de $\mathrm{Nb} 21$, la notion abstraite de 'ôt se substitue à celle, concrète, de nes.

C'est pourquoi il est parfaitement cohérent que, dans le cryptogramme chiffré par le carré « Adonaï », les lettres non utilisées pour $A R A, A E R E A$ et SERPENS forment précisément le mot $O T$ :

\begin{tabular}{|c|c|c|c|c|}
\hline $\mathrm{T}$ & $\mathrm{O}$ & & $\mathrm{O}$ & $\mathrm{T}$ \\
\hline $\mathrm{A}$ & & & & $\mathrm{A}$ \\
\hline $\mathrm{R}$ & & & & $\mathrm{R}$ \\
\hline $\mathrm{A}$ & & & & $\mathrm{A}$ \\
\hline $\mathrm{T}$ & $\mathrm{O}$ & & $\mathrm{O}$ & $\mathrm{T}$ \\
\hline
\end{tabular}

\begin{tabular}{|c|c|c|c|c|}
\hline $\mathrm{T}$ & $\mathrm{O}$ & & $\mathrm{O}$ & $\mathrm{T}$ \\
\hline $\mathrm{A}$ & $\mathrm{E}$ & $\mathrm{R}$ & $\mathrm{E}$ & $\mathrm{A}$ \\
\hline & & & & \\
\hline $\mathrm{A}$ & $\mathrm{E}$ & $\mathrm{R}$ & $\mathrm{E}$ & $\mathrm{A}$ \\
\hline $\mathrm{T}$ & $\mathrm{O}$ & & $\mathrm{O}$ & $\mathrm{T}$ \\
\hline
\end{tabular}

\begin{tabular}{|c|c|c|c|c|}
\hline T & $\mathrm{O}$ & $\mathrm{P}$ & $\mathrm{O}$ & $\mathrm{T}$ \\
\hline & $\mathrm{E}$ & $\mathrm{R}$ & $\mathrm{E}$ & \\
\hline & $\mathrm{S}$ & $\mathrm{N}$ & $\mathrm{S}$ & \\
\hline & $\mathrm{E}$ & $\mathrm{R}$ & $\mathrm{E}$ & \\
\hline $\mathrm{T}$ & $\mathrm{O}$ & $\mathrm{P}$ & $\mathrm{O}$ & $\mathrm{T}$ \\
\hline
\end{tabular}

Le SATOR, qui reproduit la «forme de l'autel de YHWH » (Jos 22), en l'occurrence celle de l'autel de bronze, est bien conçu comme un « signe et un témoin de YHWH » (Is 19,20). Précisons que le bilinguisme latin-hébreu de ARA (SERPENS) OT est attesté dans la plus importante inscription juive de Sicile, «a bilingual that has an opening formula in Hebrew and then continues in Latin ${ }^{45}$ », et à

42. Michèle Morgen, «Le Fils de l'Homme élevé en vue de la vie éternelle », loc. cit.

43. Targum du Pentateuque, t. III, éd. Roger le Déaut, Cerf, Paris, 1979, p. 194, n. 4.

44. Marcus Jastrow, Dictionary of the Targumim, Talmud Babli, Yerushalmi and Midrashic Literature, t. I, Judaica Press, New York, 1996, p. 132.

45. Leonard Victor Rutgers, «Interaction and Its limits: Some Notes on the Jews of Sicily in Late Antiquity », Zeitschrift für Papyrologie und Epigraphik, 115, 1997, p. 245-256, repris dans The Hidden Heritage of Diaspora Judaism, op. cit., p. 139-156 (p. 140). 
Pompéi même, dans une inscription prophylactique découverte en $1960^{46}$ :

\section{POINIVM CHEREM}

En outre, ce bilinguisme et la transcription latine de l'hébreu apparaissent plus près encore du SATOR, sous le carré lui-même.

\section{SAUTRAN : LA SIGNATURE HÉBRAÏQUE DU SATOR}

On considère traditionnellement les mots SAUTRAN et VALE comme le salut d'un certain Sautran(us), non sans difficulté du point de vue de l'onomastique latine, puisque $\mathrm{M}$. Della Corte suggère de lire Saturan $(u s)^{47}$. Or cette interprétation traditionnelle soulève trois objections : 1) SAUTRAN n'est pas plus proche de VALE que de $S A T O R$, dernier mot du carré ; 2) sa première lettre est alignée sur celle de $S A T O R$, alors que VALE est nettement décalé ; 3) enfin, ses lettres ont la même taille que celles de SATOR, tandis que celles de VALE, on l'a vu, sont beaucoup plus petites. Par conséquent, si SAUTRAN doit être rapproché d'un mot, c'est de SATOR.

Dès lors, une question s'impose : SAUTRAN et SATOR ne seraientils pas formés sur une même racine ? Le latin n'offre aucune solution, mais l'hébreu biblique atteste fréquemment la racine $s t r$ « cacher »: en Ex 3,6, quand Moïse cache son visage devant le buisson ardent ; à propos du sort de la Diaspora, qui fait partie des choses cachées selon Dt 29,27-28 ; pour exprimer la résistance patiente d'Israël en Is 49,2: «Il a fait de moi une flèche acérée, il m'a caché dans son carquois »; enfin, pour caractériser YHWH lui-même dans le fameux passage d'Is 45,15: "Tu es un Dieu qui se tient caché, le Dieu d'Israël, celui qui sauve ! » L'actualité de ce thème au début de l'ère chrétienne est attestée par Genèse Rabba, qui commente Dn 2,22

46. Voir Isidoro Kahn, «Un graffito recentemente scoperto a Pompei », Annuario di Studi Ebraici, 1963, p. 35-40 ; David Noy, Jewish Inscriptions of Western Europe, t. I, Cambridge, 1993, n 39 ; Giancarlo Lacerenza, « Per un riesame della presenza ebraica a Pompei », art. cit., p. 102.

47. C.I.L., t. IV, Supplément (3), Berlin, 1952, p. 902. 
( "Il dévoile les tréfonds et le caché ») dans une perspective sotériologique, en le rapprochant du Ps 31,21: «Tu caches au secret de ta face. $^{48}$ »

De fait, sachant que la lettre $N$ est une image du serpent, luimême symbolon de Dieu, et doit être considérée à part (comme le souligne ANO inscrit par la seconde main), les mots SATOR et SAUTRA correspondent respectivement au paradigme de l'infinitif absolu (qatôl) et à celui de l'impératif masculin singulier dit « emphatique » $(\text { qotlah })^{49}$. Pour ce dernier, la graphie pompéienne SAUTRA est particulièrement intéressante : elle illustre le fait que, dans le latin $\mathrm{du} \mathrm{I}^{\mathrm{er}}$ siècle, la diphtongue $A U$ est fréquemment contractée en $O$, par exemple dans le nom aurum « or », prononcé orum $^{50}$; on peut aussi le voir dans la correspondance de Cicéron, chez Horace et sur les murs mêmes de Pompéi ${ }^{51}$; enfin, une anecdote révélatrice est rapportée par Suétone ( II $^{\mathrm{e}} \mathrm{s}$.) au sujet de l'empereur Vespasien, mort le 23 juin 79, deux mois avant l'éruption du Vésuve : «Quand, un jour, l'ancien consul Mestrius Florus eut fait remarquer à Vespasien que, pour s'exprimer correctement, il fallait dire plaustra (chariots) plutôt que plostra, celui-ci le salua le lendemain en l'appelant Flaurus. ${ }^{52}$ »Ces parallèles contemporains de notre inscription montrent que SAUTRA peut sans difficulté être lu sotrah - d'autant plus que cette graphie latine concorde avec la fixation définitive de la vocalisation massorétique ( $\mathrm{IX}^{\mathrm{e}} \mathrm{s}$.) : distinct de $O$ (holem), $A U$ est apparenté à $A$ comme le qames hatouph au qames.

C'est pourquoi il est probable qu'un juif de la Diaspora, voyant dans le mot SATOR la racine str et la forme de l'infinitif absolu, aura délibérément inscrit au-dessous une autre forme de cette racine.

48. Midrach Rabba, op. cit., I, 6, p. 39.

49. Paul Joüon, Grammaire de l'hébreu biblique, Rome, 1923, 1996, p. 27 et 108 sq. Le qal de str, sans occurrence biblique, est cependant attesté dans les manuscrits de Qumran (voir le Theological Dictionary of the Old Testament, t. X, Michigan, 1999, p. 371 : «Interestingly, the niphal and hiphil here are joined by occurrences of the qal. »).

50. Max Niedermann, Phonétique historique du latin, Klincksieck, Paris, 1953 , p. 65.

51. Ibid. (C.I.L., t. IV, $\left.\mathrm{n}^{\circ} 2353\right)$.

52. Ibid., p. 66. 
Son choix de l'impératif "emphatique » a deux justifications possibles : 1) d'un point de vue syntaxique, SAUTRA constitue avec SATOR une séquence paronomastique fréquente en hébreu biblique, où l'infinitif absolu apporte une nuance emphatique en redoublant l'idée exprimée par la forme verbale conjuguée ${ }^{53}$ - la seule objection est que, quand cette forme verbale est un impératif, l'hébreu biblique postpose l'infinitif absolu de même racine ${ }^{54}$, mais l'auteur de l'inscription n'avait évidemment pas cette liberté ; 2) SAUTRA est suivi de la lettre $N$, image du serpent de $\mathrm{Nb} 21$, lui-même symbolon de Dieu dans le livre de la Sagesse et sur l'amulette répertoriée par Goodenough.

Cette analyse de SAUTRA $N$ est corroborée par l'archéologie sémitique, car le Catalogue des sceaux ouest-sémitiques de P. Bordreuil répertorie le nom ywstr, "qui relève de la racine satar "protéger", "cacher" » et « signifie que "YW a abrité", $c f$. Ps. 91,1 "Toi qui habites à l'abri (beseter) du Très-Haut" » ${ }^{55}$; mieux encore, l' Onomastica aramaica de W. Kornfeld mentionne les formes stryh et str'l, c'està-dire deux séquences du type str+nom divin $(y h \text { et ' } l)^{56}$. Or, l'impératif SAUTRA concorde parfaitement avec une telle séquence, comme le montre l'emploi des racines $s h m r$ «garder» et $z k r$ «se souvenir »; en effet, leur infinitif absolu est utilisé comme impératif ou futur injonctif dans les commandements divins du Décalogue : Dt 5,12 « observe (shamôr) le jour du Sabbat », Ex 13,3 « souvenezvous (zakôr) »; en revanche, leur impératif emphatique sert à formuler des prières: Ps 25,20 «Garde (shomrah) mon âme », 2 Ch 6,42 « Souviens-toi (zokrah) des grâces faites à David ». C'est pourquoi,

53. Paul Joüon, op. cit., p. 348 sq. ; Takamitsu Muraoka, Emphatic words and structures in biblical Hebrew, Brill, Jérusalem-Leiden, 1985, p. $91 \mathrm{sq}$. (pour la racine str, $c f$. Dt 31,18 et la séquence inf. abs. + inaccompli Hiphil).

54. Paul Joüon, loc. cit.; Takamitsu Muraoka, op. cit., p. 89.

55. Pierre Bordreuil, Catalogue des sceaux ouest-sémitiques inscrits de la Bibliothèque Nationale du Musée du Louvre, BNF, Paris, 1986, p. 54.

56. Walter Kornfeld, Onomastica aramaica aus Ägypten, Bohlaus Nachf, Vienne, 1978, p. 65 ; cf. stry'l dans Martin Noth, Die israelitischen Personennamen im Rahmen der gemeinsemitischen Namengebung, Kohlhammer, Stuttgart, 1928, p. 158. 
sous la forme d'infinitif absolu SATOR, SAUTRA $N$ est vraisemblablement une prière de forme str+nom divin : « Cache-moi, YHWH. » C'est ce qu'aura voulu souligner la seconde main juive, en répétant la lettre $N$ dans $A N O$.

\section{Conclusions}

Nombre de publications sur le SATOR concernaient l'origine et le sens du mot $A R E P O$, qui s'avère un pur accident, dû à des contraintes formelles que les auteurs du cryptogramme ne pouvaient surmonter. Cette fausse piste, parmi d'autres, a tenu les exégètes éloignés de la solution du SATOR, qui réunit deux domaines de recherche dont les points de contact sont rares : l'histoire des religions et celle des sciences. En effet, c'est un traité d'arithmétique pythagoricienne qui permet de faire le lien entre ce cryptogramme juif du $\mathrm{I}^{\mathrm{er}}$ siècle et l'opuscule byzantin du XIV qui en a consigné la clé, probablement sans le savoir - treize siècles de tradition perdue ou souterraine, comme pour le carré magique chinois (supra).

Les créateurs du SATOR, qui ont probablement vécu peu avant l'ère chrétienne, se situent dans la tradition de la Diaspora transjordanienne en Jos 22 : leur carré reproduit la « forme de l'autel de YHWH », en l'occurrence l'autel de bronze d'Ex 27, et ce «non en vue d'holocaustes ou d'autres sacrifices, mais comme un témoin »; ce signe de reconnaissance s'inscrit en outre dans la perspective sotériologique d'Is 19,19-20 : "Ce jour-là, il y aura un autel dédié à YHWH au milieu du pays d'Égypte [...]. Ce sera un signe et un témoin de YHWH Sabaot. » De fait, chiffré par le carré « Adonaï », le SATOR est ARA AEREA par ses dimensions, SERPENS en tant que palindrome, mais aussi par sa lecture boustrophédon (à l'image du serpentis lusus pompéien), et SERPENS AEREA parce qu'il n'a ni début ni fin. C'est pourquoi il est aussi OT, conformément à $\mathrm{Nb} 17$, où Dieu fait du bronze de l'autel un 'ôt pour Israël, et conformément au Targum Onkelos de $\mathrm{Nb} 21$, qui associe ' $t$ t au serpent de bronze. La correspondance entre le fond et la forme de l'inscription est fondamentale : de même que l'incipit serpentis lusus prouve que le 
calligramme dessine délibérément un serpent, ARA AEREA prouve que les dimensions du SATOR reproduisent délibérément celles de l'autel de bronze.

En revanche, même si le jeu sur la racine hébraïque str concorde parfaitement avec le caractère cryptographique du palindrome, il est difficile de dire s'il remonte à sa création première : c'est peutêtre une relecture personnelle de celui qui l'a gravé sur la palestre - et qui a fort peu de chances d'avoir aussi créé le cryptogramme. En tout cas, le SATOR est une création juive, et celui de la palestre pompéienne est aussi juif ; quant à ceux inscrits à Budapest ( $\mathrm{II}^{\mathrm{e}} \mathrm{s}$.), Circenster ou Doura-Europos (III ${ }^{\mathrm{e}} \mathrm{s}$.), rien ne permet de décider si leurs auteurs étaient juifs ou chrétiens, mais on peut penser que le judaïsme aura délaissé très tôt ce cryptogramme en latin, pour les mêmes raisons qu'il a finalement abandonné la version grecque des Septante, devenue la Bible des chrétiens.

nicolas.vinel@free.fr 20 rue des Rasselins 75020 Paris 

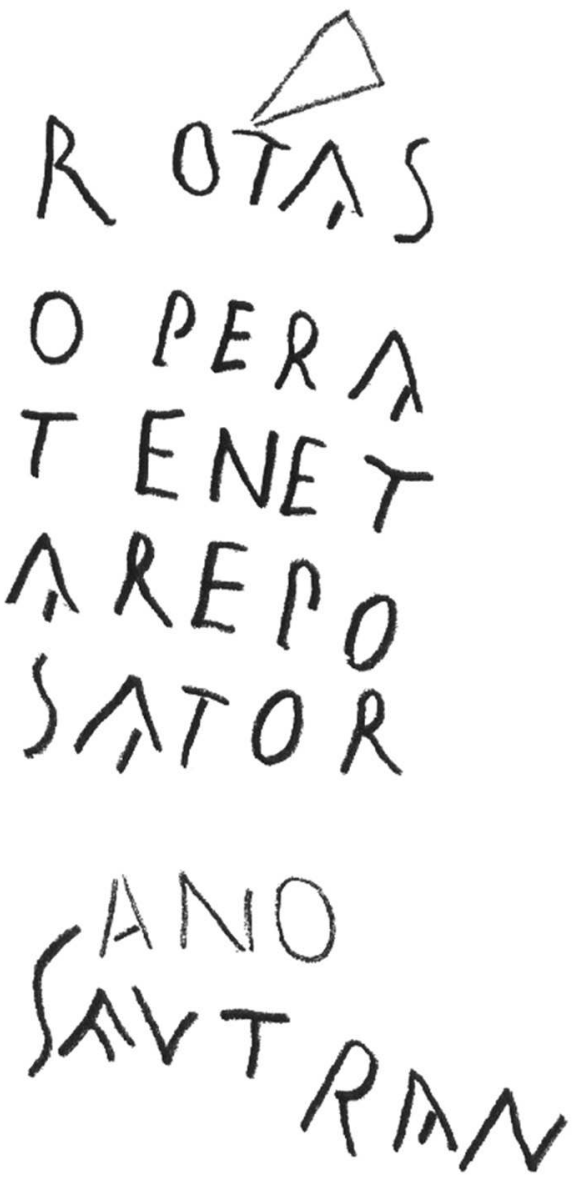

\section{$V \lambda L F$}

Le SATOR de la palestre pompéienne, d'après la photographie publiée en 1936 par M. Della Corte, art. cit., (n. 2). 